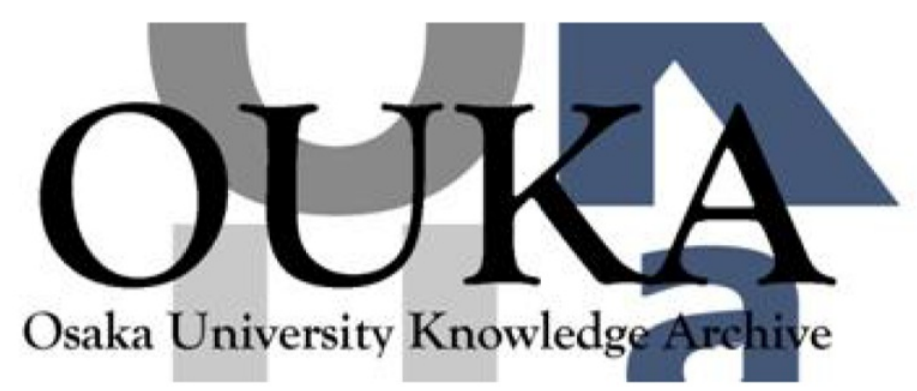

\begin{tabular}{|c|l|}
\hline Title & $\begin{array}{l}\text { Ultralow-Power Current Reference Circuit with } \\
\text { Low Temperature Dependence }\end{array}$ \\
\hline Author(s) & $\begin{array}{l}\text { Hirose, Tetsuya; Matsuoka, Toshimasa; } \\
\text { Taniguchi, Kenj i et al. }\end{array}$ \\
\hline Citation & $\begin{array}{l}\text { IEICE Transactions on Electronics. E88-C(6) } \\
\text { p.1142-p. 1147 }\end{array}$ \\
\hline Issue Date & $2005-06$ \\
\hline oaire:version & VoR \\
\hline URL & https://hdl. handle. net/11094/51720 \\
\hline rights & copyright $\odot 2005$ IEICE \\
\hline Note & \\
\hline
\end{tabular}

Osaka University Knowledge Archive : OUKA

https://ir. Library. osaka-u. ac. jp/

Osaka University 


\title{
Ultralow-Power Current Reference Circuit with Low Temperature Dependence
}

\author{
Tetsuya HIROSE $^{\dagger a)}$, Toshimasa MATSUOKA ${ }^{\dagger \dagger}$, Kenji TANIGUCHI ${ }^{\dagger \dagger}$, Tetsuya ASAI ${ }^{\dagger}$, \\ and Yoshihito AMEMIYA ${ }^{\dagger}$, Members
}

\begin{abstract}
SUMMARY An ultralow power constant reference current circuit with low temperature dependence for micropower electronic applications is proposed in this paper. This circuit consists of a constant-current subcircuit and a bias-voltage subcircuits, and it compensates for the temperature characteristics of mobility $\mu$, thermal voltage $V_{T}$, and threshold voltage $V_{T H}$ in such a way that the reference current has small temperature dependence. A SPICE simulation demonstrated that reference current and total power dissipation is $97.7 \mathrm{nA}, 1.1 \mu \mathrm{W}$, respectively, and the variation in the reference current can be kept very small within $\pm 4 \%$ in a temperature range from -20 to $100^{\circ} \mathrm{C}$.

key words: CMOS, reference, subthreshold, weak inversion, low power, temperature dependence
\end{abstract}

\section{Introduction}

One of the promising areas of research in microelectronics is the development of ultralow power analog LSIs that consist of subthreshold MOSFETs, or MOSFETs that are operated in the region of weak inversion. To construct such LSIs, we must first develop voltage and current references that can operate with low-power dissipation of a few micro watts or less. We herein describe one such low power reference, a current reference that uses a subthreshold MOSFET circuit and that generates a constant current independent of temperature and supply voltage.

In the near future, "ubiquitous" network systems will be created. Such systems require many sensing LSIs, or smart sensors, that measure various physical data in surroundings, that store the measured data, and that output the data on demand. These sensing LSIs must operate with a low-power supply because they will probably be placed under conditions where they have to get the necessary energy from microbatteries or from less-than-ideal surroundings such as ones with poor sunshine, weak electric waves, and slight differences in temperature between day and night. To create an ultralow power LSIs, we designed a circuit in the subthreshold region. In such an LSI operated in the subthreshold region, we need to develop a constant voltage supply circuit and a constant current reference circuit that has ultralow power dissipation for these LSIs.

\footnotetext{
Manuscript received November 8, 2004.

Manuscript revised January 14, 2005.

†The authors are with the Department of Electrical Engineering, Hokkaido University, Sappro-shi, 060-8628 Japan.

${ }^{\dagger \dagger}$ The authors are with the Department of Electronics and Information Systems, Osaka University, Suita-shi, 565-0871 Japan.

a) E-mail: hirose@ sapiens-ei.eng.hokudai.ac.jp

DOI: $10.1093 /$ ietele/e88-c.6.1142
}

Many current reference circuits operating with strong inversion has been reported [1]. A Beta Multiplier Self Biasing circuit is widely used as a current reference circuit using MOSFET. However, this circuit requires a very large value of the resistor to generate a small output current, and moreover, has a problem in which the output current increases linearly as the temperature increases. A current reference circuit reported by Oguey and Aebischer [2] generates a small output current using strong inversion transistors that operate in the linear and saturation regions. Resistors are not used. However, the circuit is not enough for the discussion on the temperature dependence on its output reference current. The circuit is not enough to use as a reference current under an environment where the temperature changes because the output current that they proposed increases proportionally as the temperature increases (see Appendix).

There are few reports on the premise of operating in the subthreshold region. The purpose of this work is to offer a design method for a constant current reference circuit that works at the subthreshold region over a large temperature range. This paper is organized as follows: circuit configurations and operation principles are described in Sect. 2, simulation results are presented in Sect. 3, and we conclude the paper in Sect. 4. Note that all the simulations were performed using SPICE BSIM3 level 49 model and $0.25 \mu \mathrm{m}$ TSMC-CMOS parameters.

\section{Circuit Configuration}

The current reference circuit we describe consists of a constant-current subcircuit and a bias-voltage subcircuit. The latter supplies a bias voltage to the former.

\subsection{Constant-Current Subcircuit}

Figure 1(a) shows the constant current subcircuit. It is based on the $\beta$-multiplier self biasing circuit (Fig. 1(b)) and uses a MOS resistor $M_{R}$ instead of an ordinary resistor $\mathrm{R}$. We operate all MOSFETs in this circuit in the weak-inversion region except for the MOS resistor in the strong-inversion and triode region. This circuit has two advantages compared with the basic $\beta$ multiplier: the first is that it needs no resistor of high resistance that occupies a large area on an LSI chip, and the second is that it can achieve a zero temperature coefficient of current for an appropriate bias voltage $V_{B I A S}$ for the MOS resistor. This circuit works as follows. 
(a)

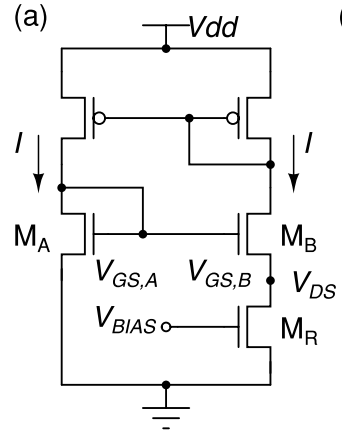

(b)

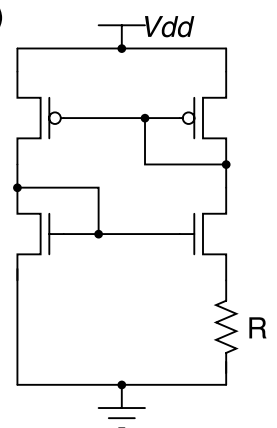

Fig. 1 Constant-current subcircuit (a) configuration of the circuit we use, and (b) basic $\beta$-multiplier self biasing circuit.

The subthreshold MOS current [3], [4] for a drainsource voltage $V_{D S}$ higher than $0.1 \mathrm{~V}$ is given by

$$
\begin{aligned}
& I=K I_{0} \exp \left(\frac{V_{G S}-V_{T H}-V_{O F F}}{\eta V_{T}}\right) \\
& I_{0}=\mu C_{O X} V_{T}^{2}(\eta-1),
\end{aligned}
$$

where $K$ is the aspect ratio (=channel width/channel length), $\mu$ is the mobility, $C_{O X}$ is the gate-oxide capacitance, $V_{T}$ is the thermal voltage $\left(V_{T}=k_{B} T / q\right), \eta$ is the subthreshold swing parameter, $V_{T H}$ is the threshold voltage, $V_{O F F}$ is the offset voltage difference between the threshold voltage in the strong inversion and that in the subthreshold region, and $I_{0}$ is called the pre-exponential factor. Voltage $V_{O F F}$ depends on the channel width and can be given by

$$
V_{O F F}=V_{O F F, 0}+\frac{V_{O F F, W}}{W},
$$

where $V_{O F F, 0}$ is the large-channel-width offset voltage, $V_{O F F, W}$ is a positive coefficient, and $W$ is the channel width.

In the circuit in Fig. 1(a), the gate-source voltage $V_{G S, A}$ in transistor $\mathrm{M}_{\mathrm{A}}$ must be equal to the sum of the gate-source voltage $V_{G S, B}$ in $\mathrm{M}_{\mathrm{B}}$ and the drain-source voltage $V_{D S}$ in $\mathrm{M}_{\mathrm{R}}$, or

$$
V_{G S, A}=V_{G S, B}+V_{D S} .
$$

Because the currents $I$ through the transistors $\mathrm{M}_{\mathrm{A}}$ and $\mathrm{M}_{\mathrm{B}}$ are the same, Eq. (3) can be rewritten as

$$
V_{D S}=\alpha_{A, B}+\eta V_{T} \ln \left(\frac{K_{B}}{K_{A}}\right),
$$

where $\alpha_{A, B}\left(=V_{O F F, A}-V_{O F F, B}\right)$ is the difference in the offset voltages, and $K_{A}$ and $K_{B}$ are the aspect ratios of transistors $\mathrm{M}_{\mathrm{A}}$ and $\mathrm{M}_{\mathrm{B}}$. The resistance $R_{M_{R}}$ of MOSFET $\mathrm{M}_{\mathrm{R}}$ operated in the triode region is given by

$$
R_{M_{R}}=\frac{1}{\mu C_{O X}\left(\frac{W}{L}\right)\left(V_{B I A S}-V_{T H}\right)} .
$$

We find from Eqs.(1),(2),(4), and (5) that the current $I$ through $\mathrm{M}_{\mathrm{A}}$ and $\mathrm{M}_{\mathrm{B}}$ is given by

$$
\begin{aligned}
I=\frac{V_{D S}}{R_{M_{R}}}= & \left(\alpha_{A, B}+\eta V_{T} \ln \left(\frac{K_{B}}{K_{A}}\right)\right) \\
& \times \mu C_{O X}\left(\frac{W}{L}\right)\left(V_{B I A S}-V_{T H}\right) .
\end{aligned}
$$

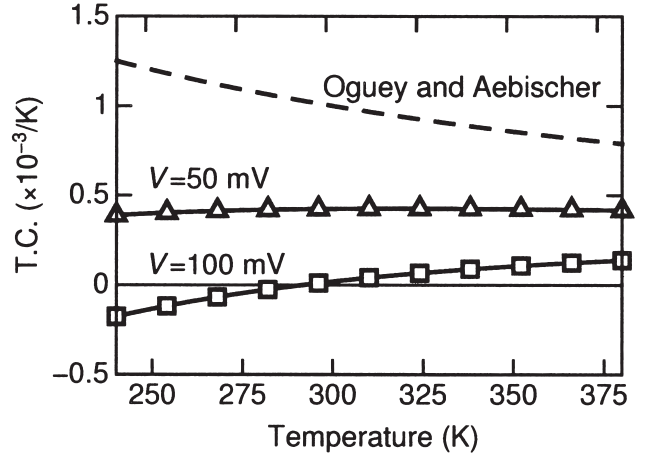

Fig. 2 Value of TC as a function of temperature, calculated from Eq. (10), with the overdriving voltage $V$ as a parameter. The dashed curve shows the TC of Oguey and Aebischer's circuit.

\subsection{Temperature Dependence of the Circuit Current}

Equation (6) shows that the temperature coefficient (TC) of current $I$ is

$$
\begin{aligned}
T C_{I} & =\frac{1}{I} \frac{\partial I}{\partial T} \\
& =\frac{1}{\alpha_{A, B}+\eta V_{T} \ln \left(\frac{K_{B}}{K_{A}}\right)} \frac{\partial\left(\alpha_{A, B}+\eta V_{T} \ln \left(\frac{K_{B}}{K_{A}}\right)\right)}{\partial T} \\
& +\frac{1}{\mu} \frac{\partial \mu}{\partial T}+\frac{1}{V_{B I A S}-V_{T H}} \frac{\partial\left(V_{B I A S}-V_{T H}\right)}{\partial T} .
\end{aligned}
$$

The temperature dependence on the mobility and threshold voltage are

$$
\begin{aligned}
& \mu=\mu\left(T_{0}\right)\left(\frac{T}{T_{0}}\right)^{-m}, \\
& V_{T H}=V_{T H 0}-\kappa T,
\end{aligned}
$$

where $\mu\left(T_{0}\right)$ is the mobility at room temperature $\left(T_{0}\right), V_{T H 0}$ is the threshold voltage at absolute zero, and $\kappa$ is the temperature dependence parameter. For a fixed reference voltage $V_{B I A S}$, TC can be expressed by equation

$$
\begin{aligned}
T C_{I}= & \frac{\eta V_{T} \ln \left(\frac{K_{B}}{K_{A}}\right)}{T\left(\alpha_{A, B}+\eta V_{T} \ln \left(\frac{K_{B}}{K_{A}}\right)\right)} \\
& -\frac{m}{T}+\frac{\kappa}{V+\kappa T},
\end{aligned}
$$

where $V\left(=V_{B I A S}-V_{T H 0}\right)$ is the overdriving voltage. The first term on the right is almost equal to $1 / T$ for small $\alpha_{A, B}$ values, the second term is about $-1.5 / T$ for ordinary MOSFETs, and the third term changes from $1 / T$ to 0 as a function of $V$. Therefore, TC can be set to 0 at room temperature by adjusting $V$ to an appropriate value. Figure 2 shows the calculated value of $\mathrm{TC}$ as a function of temperature. In this example, a zero TC can be obtained at $V=100 \mathrm{mV}$ at room temperature. The value of TC for Oguey and Aebischer's circuit, calculated from Eq. (A-3), is also depicted for comparison. 


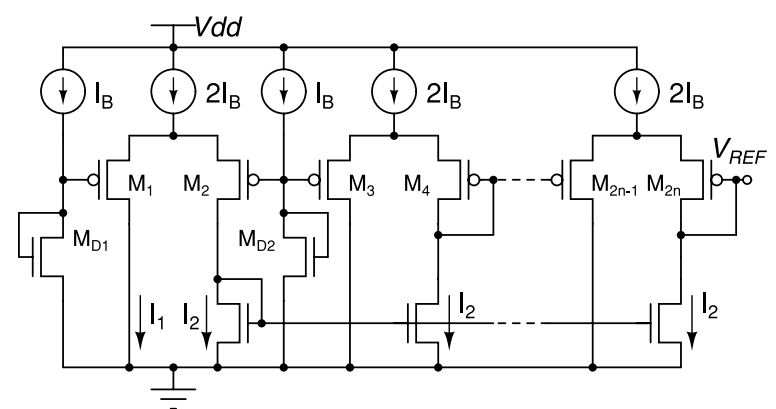

Fig. 3 Bias voltage circuit. It consists of two diode-connected transistors and $n$ differential pairs.

\subsection{Bias-Voltage Subcircuit}

The next part is to construct a subcircuit that supplies bias voltage $V_{B I A S}$ to the constant-current subcircuit. This biasvoltage subcircuit must generate a constant voltage that is independent of temperature. Although a bandgap reference circuit [5] is widely used to obtain a constant voltage, it is not suitable for our purpose because, for low-current operation in the subthreshold region, it needs large resistors of high resistance. However, Buck and others proposed a new CMOS bandgap reference circuit without resistors [6]. We modified their circuit to operate in the subthreshold region and to generate a bias voltage for the constant-current subcircuit.

Figure 3 shows the circuit configuration. The circuit consists of two diode-connected transistors $\left(\mathrm{M}_{\mathrm{D} 1}, \mathrm{M}_{\mathrm{D} 2}\right)$ and $n$ differential pairs $\left(\mathrm{M}_{1}-\mathrm{M}_{2}, \mathrm{M}_{3}-\mathrm{M}_{4}, \cdots, \mathrm{M}_{2 n-1}-\mathrm{M}_{2 n}\right)$ connected in a cascade. We set the aspect ratios of transistors such that

$$
\begin{aligned}
& K_{D 1}>K_{D 2}, \\
& K_{1}>K_{2}, \\
& \text { and } K_{2 i}>K_{2 i-1} \quad(i=2,3, \cdots, n) .
\end{aligned}
$$

The circuit produces a constant voltage on the output terminal $V_{R E F}$. The output voltage is equal to the sum of the voltage across $\mathrm{M}_{\mathrm{D} 2}$ and the gate-to-gate voltages of the differential pairs. As shown later, the $\mathrm{M}_{\mathrm{D} 2}$ voltage shows a negative TC, whereas the gate-to-gate voltages show a positive TC; this enables us to generate a zero-TC voltage. The details of the circuit operation are as follows.

The diode-connected MOSFETs $\mathrm{M}_{\mathrm{D} 1}$ and $\mathrm{M}_{\mathrm{D} 2}$ are operated in the subthreshold region, and their gate-source voltages $V_{G S, D 1}$ and $V_{G S, D 2}$ are given by

$$
\begin{array}{r}
V_{G S, D i}=V_{T H}+V_{O F F, D i}+\eta V_{T} \ln \left(\frac{I_{B}}{K_{D i} I_{0}}\right) \\
(i=1,2),
\end{array}
$$

where $I_{B}$ is the current through the MOSFETs. The temperature coefficient of this gate-source voltage is given by

$$
\frac{\partial V_{G S, D i}}{\partial T}=\frac{\partial V_{T H}}{\partial T}+\frac{\eta V_{T}}{T} \ln \left(\frac{I_{B}}{K_{D i} I_{0}}\right)
$$
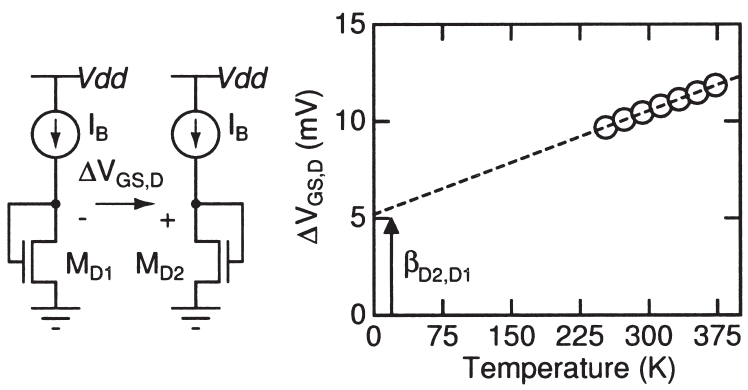

Fig. 4 Difference in gate-source voltage in two diode-connected transistors. The left portion is the schematic, and the right portion is the simulation results. The bias current $I_{B}$ and the ratio of $K_{D 1} / K_{D 2}$ are set to $100 \mathrm{nA}$ and 1.25 .

$$
\begin{aligned}
& -\eta V_{T}\left(\frac{1}{\mu} \frac{\partial \mu}{\partial T}+\frac{1}{V_{T}^{2}} \frac{\partial V_{T}^{2}}{\partial T}\right) \\
= & -\kappa+\frac{\eta k_{B}}{q}\left(\ln \left(\frac{I_{B}}{K_{D i} I_{0}}\right)-(2-m)\right) .
\end{aligned}
$$

This equation includes $I_{0}$, but the temperature dependence of $I_{0}$ can be ignored because $I_{0}$ is contained in a logarithmic function. For ordinary MOSFET parameters, the gatesource voltage across $\mathrm{M}_{\mathrm{D} 2}$ shows a negative $\mathrm{TC}$.

The difference $\Delta V_{G S, D}$ of the gate-source voltages in $\mathrm{M}_{\mathrm{D} 1}$ and $\mathrm{M}_{\mathrm{D} 2}$ can be expressed as

$$
\begin{aligned}
\Delta V_{G S, D} & =V_{G S, D 2}-V_{G S, D 1} \\
& =\beta_{D 2, D 1}+\eta V_{T} \ln \left(\frac{K_{D 1}}{K_{D 2}}\right),
\end{aligned}
$$

where $\beta_{D 2, D 1}\left(=V_{O F F, D 2}-V_{O F F, D 1}\right)$ is the positive offset voltage as shown in Fig. 4. This voltage difference is applied to the left differential pair $\mathrm{M}_{1}-\mathrm{M}_{2}$. The resultant currents $I_{1}$ and $I_{2}$ in the differential pair are given by

$$
I_{i}=K_{1} I_{0} \exp \left(\frac{V_{G S, D i}-V_{S}-V_{O F F, i}-V_{T H}}{\eta V_{T}}\right),
$$

where $V_{S}$ is the common source node voltage of the differential pair. Current $I_{2}$ is copied into $\mathrm{M}_{4}$ of the next differential pair. Because the currents in transistors $\mathrm{M}_{3}$ and $\mathrm{M}_{4}$ are $I_{1}$ and $I_{2}$, the gate-source voltages $V_{G S, 3}$ and $V_{G S, 4}$ are given by

$$
V_{G S, 3}=V_{T H}+V_{O F F, 3}+\eta V_{T} \ln \left(\frac{I_{1}}{K_{3} I_{0}}\right)
$$

and

$$
V_{G S, 4}=V_{T H}+V_{O F F, 4}+\eta V_{T} \ln \left(\frac{I_{2}}{K_{4} I_{0}}\right) .
$$

The gate-to-gate voltage $\Delta V_{G G}$, or the difference of the gatesource voltages $\left(\Delta V_{G G}=V_{G S, 3}-V_{G S, 4}\right)$ in the differential pair $\mathrm{M}_{3}-\mathrm{M}_{4}$ is given by

$$
\begin{aligned}
\Delta V_{G G} & =\beta_{3,4}+\eta V_{T} \ln \left(\frac{K_{4} I_{1}}{K_{3} I_{2}}\right) \\
& =\beta+\eta V_{T} \ln \left(\frac{K_{4} K_{1} K_{D 2}}{K_{3} K_{2} K_{D 1}}\right),
\end{aligned}
$$


where

$$
\beta=\beta_{D 2, D 1}+\beta_{2,1}+\beta_{3,4} .
$$

We set the aspect ratios such that

$$
K_{4} K_{1} K_{D 2}>K_{3} K_{2} K_{D 1}
$$

then $\Delta V_{G G}$ shows a positive TC. In the same way, the successive differential pairs generate their gate-to-gate voltages. These voltages are added together in the array of the differential pairs. The output of the circuit is the sum of $V_{G S, D 2}$ and the total of the gate-to-gate voltages. To produce enough output voltage to drive the MOS resistor in the strong-inversion region, we used a number of differential pairs. The output voltage of the circuit can be given by

$$
V_{R E F}=V_{G S, D 2}+(n-1) \Delta V_{G G} .
$$

We can obtain a constant voltage with a zero TC, adjusting the size of transistors and the number of differential pairs.

Figure 5 shows the simulated results for the output voltage of the circuit. In the simulation, the ratio of each of the transistor pairs shown in Table 1 was assumed, and the number of differential pairs was set to 5 (i.e., $n=5$ ). An almost

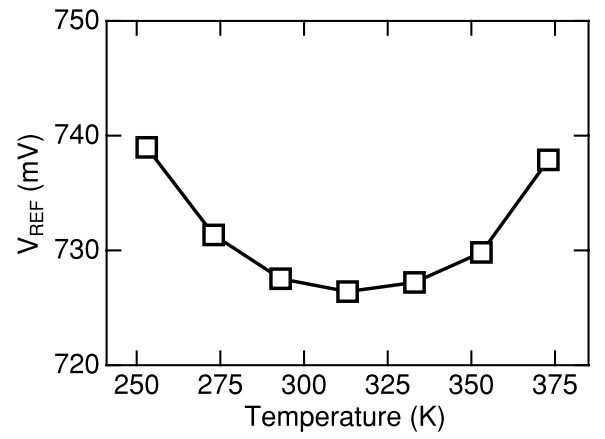

Fig. 5 Output of bias-voltage circuit as a function of temperature, simulated with parameters given in Table 1.

Table 1 Example of MOSFET's ratios.

\begin{tabular}{|c|c|c|c|}
\hline$\frac{K_{B}}{K_{A}}$ & $\frac{K_{D 1}}{K_{D 2}}$ & $\frac{K_{1}}{K_{2}}$ & $\frac{K_{i+1}}{K_{i}}(\mathrm{i}=3,5,7,9)$ \\
\hline 1.06 & 1.25 & 3 & 3 \\
\hline
\end{tabular}

constant voltage can be obtained in the temperature range, and the TC of the voltage is zero at room temperature. The voltage variation with the temperature can be suppressed within $\pm 1 \%$. This variation is mainly ascribed to the nonlinearity of the gate-source voltages of the diode-connected transistors. Though both voltages of $V_{G S, D 2}$ and $\Delta V_{G G}$ in Eq. (21), where $V_{G S, D 2}$ is negative and $\Delta V_{G G}$ is positive, are nearly proportional to the temperature, they still have little nonlinear temperature dependence. Therefore, the output voltage changes nonlinearly as shown in Fig. 5.

\section{Constructing the Current Reference Circuit}

The current reference circuit we describe can be constructed by combining the constant current subcircuit and the constant voltage subcircuit. Figure 6 shows the entire configuration of the circuit. We modified the configuration of the constant-current subcircuit and added a source-coupled amplifier, to ensure the same current flowing in $M_{A}$ and $M_{B}$. To use the source-coupled amplifier, we adapted a cascode configuration of nMOSFETs in the constant-current subcircuit. The source-coupled amplifier and the constant-current subcircuit construct the unity gain configuration. The voltage of $V_{A}$ is monitored by the non-inverting terminal of the unity gain circuit, and then the output voltage of $V_{B}$ is set at the same voltage with $V_{A}$. This ensures the same current value in transistors $\mathrm{M}_{\mathrm{A}}$ and $\mathrm{M}_{\mathrm{B}}$. $\mathrm{AC}$ analysis of this unity gain circuit shows that the phase margin at the unity gain frequency is 45 degree, and the circuit is stable.

Figure 7 shows the simulated results for the output current $I_{R E F}$. In this simulation, the ratio of $K_{B} / K_{A}$ was set to 1.06. The variation in the current can be suppressed within $\pm 4 \%$ in the temperature range from -20 to $100^{\circ} \mathrm{C}$.

The circuit is insensitive to a change in power supply voltage because of the feedback control with the sourcecoupled amplifier. Figure 8 shows the bias voltage $V_{R E F}$ and the output current as a function of the supply voltage. The circuit can operate at a low power voltage of $1.2 \mathrm{~V}$. The variation in the bias voltage and the output current can be suppressed within $\pm 0.19 \%$ and $\pm 0.60 \%$, respectively, in the supply voltage range from 1.2 to $3 \mathrm{~V}$. Table 2 summarizes the performance of this current reference.

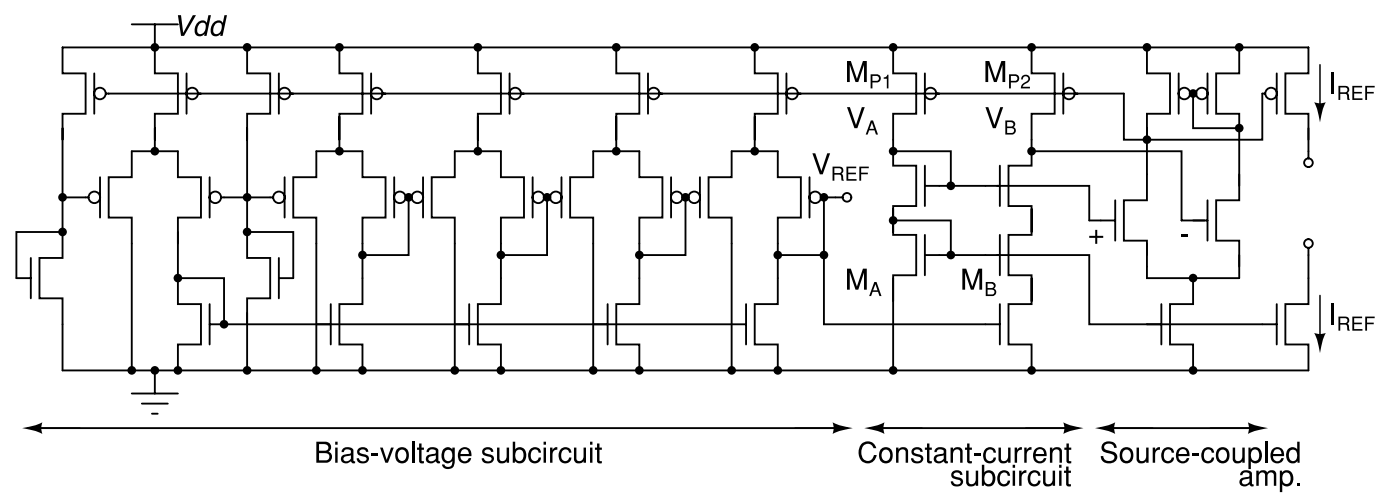

Fig. 6 Current reference circuit consisting of bias voltage subcircuit and constant-current subcircuit. 


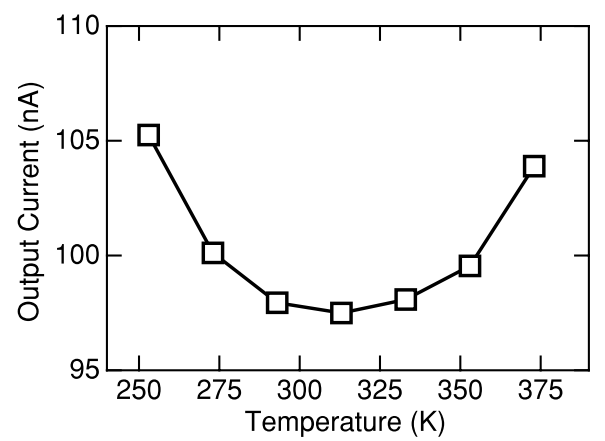

Fig. 7 Output of current reference circuit as a function of temperature, simulated with parameters given in Table 1.

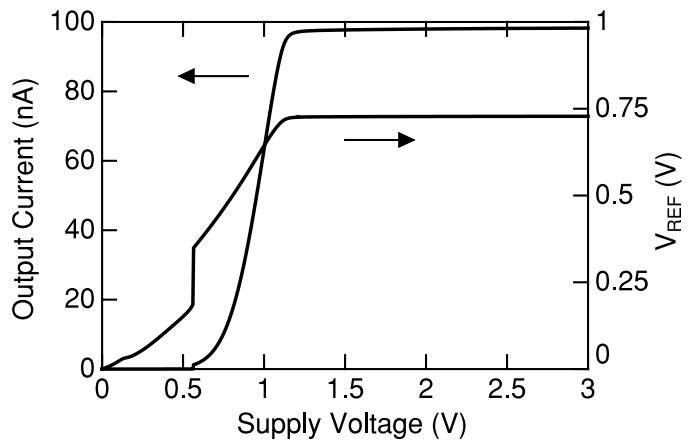

Fig. 8 Bias-voltage and output current as a function of supply voltage.

Table 2 Performance summary.

\begin{tabular}{l|l}
\hline Technology & $0.25 \mu \mathrm{m}, 1$-poly 5-metal CMOS \\
$\mathrm{V}_{D D}$ & $1.5 \mathrm{~V}$ \\
$I_{R E F}$ & $97.7 \mathrm{nA}\left(T=25^{\circ} \mathrm{C}\right)$ \\
$V_{R E F}$ & $727 \mathrm{mV}\left(T=25^{\circ} \mathrm{C}\right)$ \\
Power & $1.1 \mu \mathrm{W}\left(T=25^{\circ} \mathrm{C}\right)$ \\
$\Delta I_{R E F} / I_{R E F}$ & $\pm 4 \%\left(T=-20-100^{\circ} \mathrm{C}\right)$ \\
& $\pm 0.60 \%\left(V_{D D}=1.2-3 \mathrm{~V}\right)$ \\
$\Delta V_{R E F} / V_{R E F}$ & $\pm 1 \%\left(T=-20-100^{\circ} \mathrm{C}\right)$ \\
& $\pm 0.19 \%\left(V_{D D}=1.2-3 \mathrm{~V}\right)$ \\
\hline
\end{tabular}

The temperature dependence on the output current is somewhat larger than expected from Eq. (10). This was mainly caused by the variation in the bias-voltage with temperature that is shown in Fig. 5. Further improvements will be made possible by modifying the bias-voltage subcircuit. Equation (10) shows that the temperature that gives a zero TC increases as overdriving voltage $V$ increases (see Fig. 2). Therefore, if we can construct a bias-voltage circuit that produces a voltage with an appropriate positive TC, then we will be able to create an improved current-reference circuit that shows a zero TC over a wide temperate range. We are now developing such a modified bias-voltage circuit.

\section{Conclusion}

We described an ultralow power constant current reference circuit with little temperature dependence. This circuit consists of a constant-current subcircuit and a bias-voltage subcircuit. and is useful as a reference current for a microp- ower application in an environment where the temperature changes. This circuit compensates for the temperature characteristics of mobility $\mu$, thermal voltage $V_{T}$, and threshold voltage $V_{T H}$ in such a way that the reference current has small temperature dependence. A SPICE simulation demonstrated that the reference current is $97.7 \mathrm{nA}$, and the variation in the reference current and the bias voltage can be kept very small within $\pm 4 \%$ and $\pm 1 \%$ in a temperature range from -20 to $100^{\circ} \mathrm{C}$. The total power dissipation is extremely low, $1.1 \mu \mathrm{W}$. Standard CMOS technology can be used without a resistor.

\section{Acknowledgment}

The chip design in this work is supported by VLSI Design and Education Center (VDEC), the University of Tokyo in collaboration with Cadence Design System.

\section{References}

[1] D.A. Johns and K. Martin, Analog Integrated Circuit Design, John Wiley \& Sons, 1997.

[2] H.J. Oguey and D. Aebischer, "CMOS current reference without resistance," IEEE J. Solid-State Circuits, vol.32, no.7, pp.1132-1135, July 1997.

[3] W. Liu, X. Jin, J. Chen, M.C. Jeng, Z. Liu, Y. Cheng, K. Chen, M. Chan, K. Hui, J. Huang, R. Tu, P.K. Ko, and C. Hu, BSIM3v3.2.2 MOSFET Model-User's Manual, University of California, Berkeley, 1999.

[4] Y. Cheng, M.C. Jeng, Z. Liu, J. Huang, M. Chan, K. Chen, P.K. Ko, and $\mathrm{C}$. Hu, "A physical and scalable I-V model in BSIM3v3 for ana$\log$ /digital circuit simulation," IEEE Trans. Electron Devices, vol.44, no.2, pp.277-287, Feb. 1997.

[5] P.E. Allen and D.R. Holberg, CMOS Analog Circuit Design, New York Oxford University Press, 2002.

[6] A.E. Buck, C.L. McDonald, S.H. Lewis, and T.R. Viswanathan, "A CMOS bandgap reference without resistors," IEEE J. Solid-State Circuits, vol.37, no.1, pp.81-83, Jan. 2002.

\section{Appendix: Temperature Coefficient of Oguey and Ae- bicher's Current Reference}

The output current in their circuit [2] is given by

$$
I=n^{2} \mu C_{O X} K V_{T}^{2} K_{e f f},
$$

where $n$ is a correction factor of low drain-source voltage $\left(n=\beta_{\text {lin }} / \beta_{\text {sat }}\right)$, and $K_{\text {eff }}$ is a function of the geometrical ratios of transistors. The TC is given by

$$
T C_{I}=\frac{1}{I} \frac{d I}{d T}=\frac{1}{\mu} \frac{d \mu}{d T}+\frac{1}{V_{T}^{2}} \frac{d V_{T}^{2}}{d T}
$$

and is reduced to

$$
T C_{I}=-\frac{m}{T}+\frac{2}{T} \text {. }
$$

Because the value of $m$ is about 1.5 for ordinary MOSFETs, the temperature coefficient is always positive, and the current will increase as the temperature increases. 


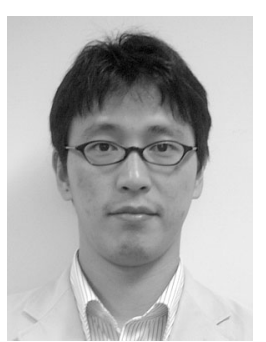

Tetsuya Hirose was born in Tottori, Japan in 1976. He received the B.S. and M.S. degrees from Osaka University, Osaka, Japan in 2000 and 2002, respectively. He is now a research associate in the Department of Electrical Engineering, Hokkaido University, Sapporo, Japan. His current research interests are in low-power analog CMOS circuits for intelligent sensors.

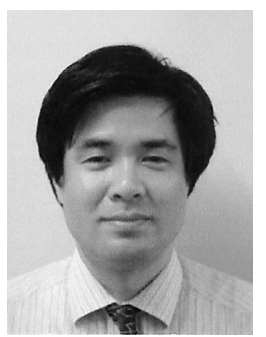

Toshimasa Matsuoka received the B.S., M.S. and Ph.D. degrees in electronic engineering from Osaka University, Osaka, Japan, in 1989, 1991 and 1996, respectively. During 1991-1998, he worked for the Central Research Laboratories, Sharp Corporation, Nara, Japan, where he was engaged in the research and development of deep submicron CMOS devices and ultra thin gate oxides. Presently, he is a Associate Professor of Electronics Engineering at Osaka University. His current research includes CMOS RF circuits and analog circuits. Dr. Matsuoka is a member of the Japan Society of Applied Physics and the IEEE.

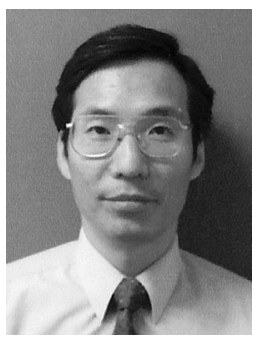

Kenji Taniguchi received the B.S., M.S. and Ph.D. degrees from Osaka University, Osaka Japan, in 1971, 1973 and 1986, respectively. From 1973 to 1986, he worked for Toshiba Research and Development Center, Kawasaki, Japan where he was engaged in process modeling and the design of MOS LSI fabrication technology. He was Visiting Scientist at Massachusetts Institute of Technology, Cambridge, from July 1982 to November 1983. Presently, he is a Professor of Electronics Engineering at Osaka University. His current research interests are in analog circuits, radio frequency circuits, device physics and process technology. Prof. Taniguchi is a member of the Japan Society of Applied Physics. He is a fellow of the IEEE.

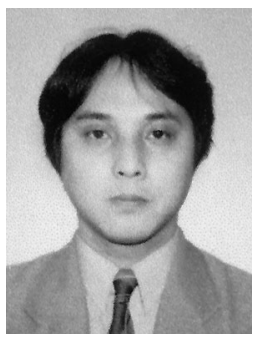

Tetsuya Asai received the B.S. and M.S. degrees in electrical engineering from Tokai University, Kanagawa, Japan, in 1993 and 1996, respectively, and the Ph.D. degree in electrical and electronic Engineering from the Toyohashi University of Technology, Aichi, Japan, in 1999. He is now an Associate Professor in the Department of Electrical Engineering, Hokkaido University, Sapporo, Japan. His current research interests include sensory information processing in neural networks as well as design and applications of neuromorphic VLSIs and the reaction-diffusion chip.

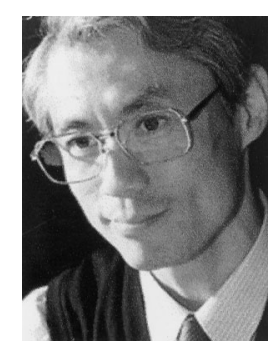

Yoshihito Amemiya received the B.S., M.S., and Ph.D. degrees from the Tokyo Institute of Technology, Tokyo, Japan, in 1970, 1972, and 1975. He joined NTT Musashino Laboratories in 1975, where he worked on the development of silicon process technologies for high-speed logic LSIs. From 1983 to 1993, he was with NTT atsugi Laboratories and developed bipolar and CMOS circuits for Boolean logic LSIs, neural network LSIs, and cellular automaton LSIs. Since 1993, he has been a Professor with the Department of Electrical Engineering, Hokkaido University, Sapporo. His research interests are in the fields of silicon LSI circuits, signal processing devices based on nonlinear analog computation, logic systems consisting of single-electron circuits, and information-processing devices making use of quantum nanostructures. 